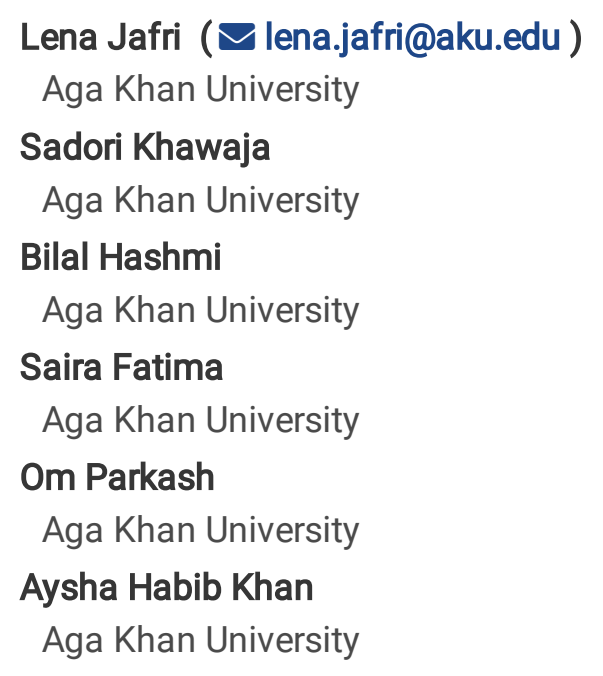

\author{
Lena Jafri ( $\sim$ lena.jafri@aku.edu ) \\ Aga Khan University \\ Sadori Khawaja \\ Aga Khan University \\ Bilal Hashmi \\ Aga Khan University \\ Saira Fatima \\ Aga Khan University \\ Om Parkash \\ Aga Khan University \\ Aysha Habib Khan \\ Aga Khan University
}

\title{
Presenting IgG4-related disease: an emerging entity in Pakistan
}

\section{Research Article}

Keywords: immunoglobulins, autoimmune diseases, pancreatitis, IgG4 related diseases, storiform fibrosis

Posted Date: April 21st, 2021

DOI: https://doi.org/10.21203/rs.3.rs-424267/v1

License: (c) (i) This work is licensed under a Creative Commons Attribution 4.0 International License. Read Full License 


\section{Abstract \\ Background}

IgG4 related disease (IgG4-RD) is a spectrum of immune mediated disorder involving various organs of the body. In this study the clinical spectrum of possible IgG4-RD was explored.

\section{Methods}

Subjects tested for serum IgG4 and all biopsies of subjects with suspected IgG4-RD received at the clinical laboratory of a tertiary care hospital from April 2015 to December 2019 were included. Medical charts of subjects registered were reviewed and telephonic interviews were conducted. Subjects were divided into two groups: group I had biochemical evidence of IgG4-RD while group II had histopathological evidence of IgG4-RD. "Comprehensive diagnostic criteria for IgG4-RD, 2011" was used for labeling patients as possible, probable and definitive IgG4-RD.

\section{Results}

A total of 177 study subjects were recruited in the current study. Group I included 10 children and 105 adults whereas group II had 5 children and 57 adults. Out of the total 177 subjects definitive, probable and possible IgG4-RD were seen in $(n=2,1.1 \%),(n=61,34.4 \%)$ and $(n=114,64.4)$ subjects respectively. The commonest organs involved in all the study subjects were pancreas (57.6\%), submandibular gland (12.4\%) and liver (6.2\%).

\section{Conclusion}

The clinical feature of IgG4-RD include single or multiple organ involvement with pancreas being the most frequently affected organ in the current population. Amalgamation of clinical, biochemical and histopathological findings are essential for the IgG4-RD, although none is pathognomonic by itself.

\section{Background}

IgG4 related disease (IgG4-RD) is a spectrum of immune mediated, chronic fibro-inflammatory disorder seen in almost every organ of the body (1). IgG4, a subclass of $\operatorname{lgG}$ is considered as diagnostic marker of this disease but around $20-40 \%$ of patients with IgG4-RD may have normal serum IgG4, despite the pathognomonic histopathological findings $(2,3)$. It is proposed that lgG4 antibodies may have some role in the inflammatory process or it may simply be produced to counter the primary inflammatory stimulus (4). The pathogenesis of IgG4-RD is not entirely understood, and role of genetic, bacterial and autoimmune factors triggering this disease has been proposed. Among the genetic factors, certain HLA serotypes such as DRB1*0405 and DQB1*0401 are associated with an increased susceptibility to IgG4-RD in Japanese populations $(5,6)$. Bacterial infections and molecular mimicry is also considered as an important initiating factor which can potentially cause the antibodies produced against bacteria to attack self-antigens $(7,8)$. Lastly, autoimmunity plays its role by initiating a Th-2 cell response, which results in the production of interleukins 4, 5, 10, and 13 and transforming growth factor $\beta$ (TGF- $\beta$ ) leading to the eosinophilia, elevated serum IgG4 and serum IgE concentrations, and progression of fibrosis that are characteristic of IgG4-RD $(4,9)$. This inflammatory process results in tumor-like enlargement of the affected sites and organ dysfunction (4).

The features classically seen on histopathological examination include a dense infiltrate of lympho-plasmocytic cells predominantly IgG4 positive plasma cells, storiform fibrosis, eosinophilic infiltrate and obliterative phlebitis $(10,11)$. The 
clinical picture and the presenting symptoms are very diverse owing to the ability of this disease to affect virtually any organ of the body $(4,10)$. There is scarcity of data about the global incidence or prevalence of IgG4-RD. Most studies are concentrated in Japan and the most frequently encountered manifestation of the disease is pancreatitis. Unlike many autoimmune diseases, the prevalence is higher in men than women. In Japan ratio of male to female patients was approximately 2.8: 1 in IgG4 related pancreatitis (12). Current study is designed to determine the clinico-pathological features of IgG4-RD in subjects with elevated serum IgG4 and in subjects with histopathological evidence of IgG4-RD being tested at the clinical laboratory.

\section{Methods}

The study was conducted between April 2015 and December 2019. The approval was sought from Aga Khan University Ethical Review Committee before initiation of the study (3871-Pat-ERC-15) before commencement of the study. All methods were performed in accordance with the relevant guidelines and regulations. All subjects tested for serum IgG4 and all biopsies of subjects with suspected IgG4-RD received at the clinical laboratory of a tertiary care hospital during this period were included. Subjects who had serum IgG4 within the reference limit were excluded from the final analysis. The study subjects were divided into two groups as follows: group I included subjects with biochemical evidence of IgG4-RD (elevated serum IgG4 levels) and group II included subjects with histopathological evidence of IgG4-RD. Subjects were interviewed over phone or through review of electronic medical records using a structured questionnaire after taking informed consent. Informed consent and history was obtained from parents/ guardians of children.

Group I-data collection and biochemical analysis: Demographics and clinical data was gathered on a structured questionnaire constituting of a detailed clinical history including symptoms, course of disease, and management, information regarding laboratory, biopsy and radiologic findings. Each subject was called multiple times and an inability to respond to 4 phone calls (days $0,1,7$ and 14) led to their exclusion from the study. Review of medical charts was also done by the investigators to gather clinical information. Serum samples stored for IgG4 estimation during this period were assayed in the Section of Chemical Pathology, Department of Pathology and Laboratory Medicine, Aga Khan University Hospital. Serum IgG4 was analyzed using Binding Site kit on Beckman-IMMAGE800 analyzer. Positive and negative controls were run with each batch of analysis. During this study period clinical laboratory successfully participated in proficiency testing surveys for serum IgG4 by College of American Pathologists. Serum IgG4 levels beyond $864 \mathrm{mg} / \mathrm{L}$ were considered elevated for subjects $>18$ years of age, for children, age appropriate cutoffs were used.

Group II-data collection and histopathological analysis: Clinical data of subjects with available biopsies was recorded on a structured questionnaire similar to Group I. All biopsies were reviewed in the Section of Histopathology, Department of Pathology and Laboratory Medicine, Aga Khan University Hospital. Biopsy findings were studied for organ involvement, histological findings like dense lymphoplasmacytic infiltrate, storiform fibrosis and the ratio of IgG4 to IgG-positive cells.

Diagnostic Criteria for IgG4-RD: Table 1 shows Components and Description of Comprehensive Diagnostic Criteria 2011, by Umehara et al used for classification of subjects. A diagnosis of definitive IgG4-RD was made if the patient had organ involvement such as organ enlargement or dysfunction based on the clinical history and radiological findings, in the presence of elevated serum IgG4 and characteristic histopathological findings. Subjects were categorized into possible IgG4-RD if they had high serum IgG4 with clinical findings and lastly a diagnosis of probable lgG4-RD was made if the patient had classic histopathological findings along with signs of organ involvement (13). Data was entered in the SPSS version 22 for analysis, pediatric (<18 years) and adult population was analyzed separately. Mean and standard deviation of quantitative data and frequency of qualitative data was derived.

\section{Results}


A total of 177 study subjects were recruited in the current study. Group I included 10 children and 105 adults whereas group II had 5 children and 57 adults. As shown in table 2 definitive, probable and possible IgG4-RD were seen in $(n=2$, $1.1 \%),(n=61,34.4 \%)$ and $(n=114,64.4)$ subjects respectively. The commonest organs involved in all the study subjects (both groups included) were pancreas $(n=102,57.6 \%)$, submandibular gland $(n=22,12.4 \%)$ and liver $(n=11,6.2 \%)$ as shown in table 3.

\section{Group I-clinical and biochemical findings}

A total of 115 subjects with high serum IgG-4 levels were included with high IgG4 levels. Ten were children and one hundred five adults were taken.

Pediatric subjects: Mean Age (SD) of children $(n=10)$ was $10.7( \pm 4.9)$ years. Majority were males $(n=7,70 \%)$. Median serum IgG4 in mg/L (IQR) was $1585 \mathrm{mg} / \mathrm{L}$ (2362.5-1295) in children. Fever was the most common complaint found in $70 \%(n=7)$ of the subjects, followed by weight-loss in $50 \%(n=5)$ of subjects, $30 \%(n=3)$ allergies, dry mouth and dry eyes and $20 \%(n=2)$ of the subjects also had arthralgia. As described in Figure 1 a the most commonly affected organ in this group was pancreas seen in all subjects $(n=7,70 \%)$.

Adult subjects: Mean age (SD) of adults $(n=105)$ included in this study was $43.8( \pm 16.4)$ years. Majority $(n=61,58 \%)$ were males. Median serum IgG4 in mg/L (IQR) was 1400.0 mg/L (2040-1095) in adults. Serum IgG4 levels were higher in pediatric population as compared to the adults in group I ( $p$ value<0.05). Out of the total one hundred and five adults in group II 104 were diagnosed with possible IgG4-RD and only one subject was diagnosed with definitive disease based on the biopsy (Table 2). The symptoms in adults were much more diverse, weight loss was seen in $60 \%(n=63)$ of subjects in this group followed by arthralgia in $39 \%$ adults $(n=41)$ and fever seen in $32.4 \%(n=34)$ as shown in Figure $1 b$. Allergies $(n=24,22.9 \%)$, dry eyes $(n=11,10.5 \%)$ and dry mouth $(n=15,14.3 \%)$ were also recorded. One patient from the total 105 adults also had ptosis. Pancreas was the most common organ involved in adults $(n=79,75.2 \%)$ as shown in table 3 . It is also important to note that $7.6 \%(n=8)$ of subjects had multiple organ involvement, which mostly pancreatitis in combination with some other disease. The radiological features seen in the subjects were diverse depending upon organ involvement. Subjects suffering from IgG4 pancreatitis, the CT scans and MRIs showed a spectrum of abnormalities ranging from showed peripancreatic fat stranding (52\%), bulky pancreas (49\%), pancreatic atrophy (21\%), and calcification of pancreas (21\%). In both the subjects with IgG4 related kidney disease imaging revealed multiple wedge shaped hypo attenuating areas in renal cortices representing renal infarcts.

\section{Group II-clinical and histopathological findings}

A total of 62 subjects with suspected IgG4-RD had their biopsies evaluated from April 2015 to December 2019.

Pediatric subjects: Five biopsy specimens received during the study period were from children with suspected IgG4-RD. Mean age (SD) of these children was $15.4( \pm 1.4)$ years three were females and two male children. The most commonly affected organ in pediatric population of group II was eyes $(n=4,80 \%)$. All five of these children were categorized as having probable IgG4-RD as per criteria defined in methodology.

Adult subjects: Fifty seven biopsy specimens received during the study period were from adults with suspected IgG4-RD. Mean age (SD) was $47.1( \pm 15.6)$ years and majority were males $(n=34,59.6 \%)$. The most commonly affected organ in adult population of group II was salivary gland $(n=17,29.8 \%)$, pancreas $(n=16,28 \%)$ and lungs $(n=7,12.3 \%)$. Out of the total fifty seven in adults of group II only one subjects had definitive IgG4-RD whereas 56 had probable IgG4-RD as per criteria defined in methodology. In the subject with definitive IgG4-RD the salivary gland biopsy revealed dense lymphoplasmacytic inflammatory infiltrate in areas showing lymphoid follicles with prominent germinal centers. These plasma cells were strongly reactive for immunoglobulin IgG4. Pan B (CD20) cell antigen and Pan T (CD3) cell antigen showed mixed pattern. Serum IgG4 were $>11000 \mathrm{mg} / \mathrm{L}$ in the same subject. 


\section{Discussion}

Out of the total $(n=177)$ subjects included majority were males $(58.7 \%)$. Overall the most commonly affected organ was pancreas $(n=102,57.6 \%)$ followed by submandibular gland $(n=23,12.9 \%)$ and liver $(n=11,6.2 \%)$. Unlike the typical demographics of autoimmune diseases, IgG4-RD is more common in males with studies reporting a ratio of 3:1 (10). Studies report that the gender distribution varies depending on the site of involvement; head and neck is seen with equal frequency in both sexes, whereas pancreas, retro-peritoneum and kidney involvement is seen more commonly in males (14). Current study displayed the same characteristics and features regardless of gender. Majority of serum IgG4 testing was in adults mainly sent by gastroenterologist. Though few but there were children with elevated serum IgG4 levels in our data. It is important for the pediatricians to suspect and recognize these disorders early and initiate therapy promptly to prevent irreversible organ damage. The causes of pancreatitis in children described in the literature are usually drugs, infections, trauma and congenital anomalies and IgG4-RD is a very rare cause of childhood pancreatitis. According to the American Academy of Pediatrics IgG4 related pancreatitis is now being increasingly recognized as an important cause, and children interestingly have type 2 autoimmune pancreatitis more commonly than adults (15). The largest case series of 48 cases of Pediatric Autoimmune pancreatitis revealed that abdominal pain (91\%) and obstructive jaundice (42\%) were the most common symptoms at diagnosis however, elevated serum IgG4 levels were only observed in $22 \%$ of these children (16). In our data there were 12 children with possible IgG4-RD and fever and weight loss was common symptom in them. Literature shows that fever and weight loss were not a common feature in children with IgG4-RD. The most commonly affected organ in pediatric population of group II was eyes $(n=4,80 \%)$. A systemic review from 2016 revealed on pediatric IgG4 RD revealed that IgG4 related orbital disease was far more common (44\%) than IgG4 related pancreatitis $(12 \%)(17)$. In adult patients enrolled in group 1 of our study, weight loss was seen in $60 \%(n=63)$ of subjects in this group followed by arthralgia in $39 \%$ adults $(n=41)$ and fever seen in $32.4 \%(n=34)$. Various studies have reported weight loss to be an important feature of IgG4-RD in adults, and on average patients lose about 10-25 lbs., but this change is not noticeable because it occurs very slowly over a long period of time (1). Adults in our study displayed a more versatile clinical picture with pancreatitis (75.2\%) and hepatitis (10.4\%) being the most common diseases in our study subjects. A Japanese group of researchers noted pancreatitis to be the most frequent diagnosis (60\%) in adult patients with IgG4-RD $(\mathrm{n}=235)$, followed by sialadenitis (34\%), IgG4 related kidney disease (23\%) and dacryoadenitis (23\%) and peri-aortitis (20\%)(18). Current study shows that $7.6 \%$ subjects with possible IgG4-RD had multiple organ involvement. The international literature reports many cases of concurrent pancreatic and renal or biliary involvement (19). In a study on patients $(n=125)$ with biopsy-proven disease at Massachusetts General Hospital Boston, multi-organ involvement was seen in $38 \%$ of the patients(20). There are two case reports on retroperitoneal fibrosis with coexistent primary biliary cholangitis $(21,22)$. Timely diagnosis of IgG4-RD, with non-specific clinical symptomatology and multi-organ multisystem involvement is challenging may require interdisciplinary cooperation of correct identification and management of this disorder. Our study also identified pancreatic tumor in 3 subjects with high IgG4 levels. Association of pancreatic cancer and IgG4-RD has been reported earlier(23) and serum IgG4 levels more than two times the normal is more likely due to IgG4- RD. Current evidence suggests there raised IgG4 levels do not seem to have any prognostic value in pancreatic cancer $(24,25)$. Another association reported is that of allergies and IgG4-RD, data suggests that up to $33 \%$ people with IgG4RD have allergies which is significantly higher than the general population (26), $22.4 \%(n=24)$ of the adult subjects and three children in our study reported allergies. Literature on IgG4-RD is scarce in Asia especially in the South-Asian region (table 4). An Indian study from India $(n=41)$ revealed ophthalmologic involvement as the commonest manifestation, seen in $13 \%$, followed by lymphadenopathy and retroperitoneal disease (27). Studies reported from far east Asia report lymph nodes, salivary and lacrimal glands as the major culprits (28). The percentage for IgG4 related Sialadenitis ranges from $33 \%$ to $79 \%(28-31)$. IgG4 related dacryoadenitis and lymphadenopathy was seen in $40-50 \%$ and $30-65 \%$ of cases with IgG4-RD (28-32). Current study has few important strengths. First, it is the only study on clinical and laboratory findings of IgG4-RD with literature review from this part of the world. Our study also compares the clinical manifestations and organ involvement in IgG4-RD in both adults and children. Although not all but some study subjects had biopsy proven IgG4-RD. There were some limitations of this study, although medical charts were reviewed but most 
information was gathered via telephonic interview. Larger multi-center study with active multidisciplinary collaboration including patients from different geographic locations from all over the world would help in greater understanding of this emerging immune-mediated systemic disease. Additionally, a head to head comparison of serum IgG4-RD and disease severity should be carried out in patients with biopsy proven IgG4-RD.

\section{Conclusion}

IgG4-RD are a spectrum of immune mediated diseases that can potentially involve any organ in the body. In our patient population, comprising of both adults and children, pancreas was the most frequently affected organ, followed by the submandibular gland and liver. Owing to the diversity of organ involvement, the symptoms can be highly non-specific and vague and require a multi-disciplinary approach to ensure timely diagnosis and treatment.

\section{Declarations}

Declaration of interest statement: Authors have no conflict of interest and no financial interests related to the subject matter of the contribution.

\section{Ethics approval and consent to participate}

Ethical approval was taken from Aga Khan University Ethical Review Committee before initiation of the study (3871-PatERC-15). Subjects were interviewed over phone after taking informed consent. Informed consent and history was obtained from parents/ guardians of children.

\section{Consent for publication}

Not applicable

\section{Availability of data and materials}

The datasets generated and/or analyzed during the current study are not publicly available due to privacy restrictions but are available from the corresponding author on reasonable request.

\section{Competing interests}

None

\section{Funding}

None

\section{Authors' contributions}

$\mathrm{LJ}, \mathrm{AHK}, \mathrm{OP}$ and SF were involved in involved in planning, conception and design of research and statistical analysis and conduct of the project. SK and BH were involved in data collection, analysis and interpretation. LJ and SK prepared the tables and figures. All authors edited, revised and approved the final version of the article.

\section{Acknowledgments}

None

\section{References}


1. Stone JH, Brito-Zerón P, Bosch X, Ramos-Casals M. Diagnostic Approach to the Complexity of IgG4-Related Disease. Mayo Clin Proc. 2015 Jul;90(7):927-39.

2. Sah RP, Chari ST. Serologic issues in IgG4-related systemic disease and autoimmune pancreatitis. Curr Opin Rheumatol. 2011 Jan;23(1):108-13.

3. Ghazale A, Chari ST, Smyrk TC, Levy MJ, Topazian MD, Takahashi N, et al. Value of serum IgG4 in the diagnosis of autoimmune pancreatitis and in distinguishing it from pancreatic cancer. Am J Gastroenterol. 2007 Aug;102(8):164653.

4. Stone JH, Zen Y, Deshpande V. IgG4-related disease. N Engl J Med. 2012 Feb 9;366(6):539-51.

5. Park DH, Kim M-H, Oh HB, Kwon O-J, Choi Y-J, Lee S-S, et al. Substitution of aspartic acid at position 57 of the DQbeta1 affects relapse of autoimmune pancreatitis. Gastroenterology. 2008 Feb;134(2):440-6.

6. Kawa S, Ota M, Yoshizawa K, Horiuchi A, Hamano H, Ochi Y, et al. HLA DRB1* 0405-DQB1* 0401 haplotype is associated with autoimmune pancreatitis in the Japanese population. Gastroenterology. 2002;122(5):1264-9.

7. Frulloni L, Lunardi C, Simone R, Dolcino M, Scattolini C, Falconi M, et al. Identification of a novel antibody associated with autoimmune pancreatitis. N Engl J Med. 2009 Nov 26;361(22):2135-42.

8. Guarneri F, Guarneri C, Benvenga S. Helicobacter pylori and autoimmune pancreatitis: role of carbonic anhydrase via molecular mimicry? J Cell Mol Med. 2005 Sep;9(3):741-4.

9. Detlefsen S, Sipos B, Zhao J, Drewes AM, Klöppel G. Autoimmune pancreatitis: expression and cellular source of profibrotic cytokines and their receptors. Am J Surg Pathol. 2008 Jul;32(7):986-95.

10. Opriţă R, Opriţă B, Berceanu D, Diaconescu IB. Overview of IgG4-related disease. Journal of medicine and life. 2017;10(4):203.

11. Saeki T, Saito A, Hiura T, Yamazaki H, Emura I, Ueno M, et al. Lymphoplasmacytic infiltration of multiple organs with immunoreactivity for IgG4: IgG4related systemic disease. Internal Medicine. 2006;45(3):163-7.

12. Nishimori I, Tamakoshi A, Otsuki M. Prevalence of autoimmune pancreatitis in Japan from a nationwide survey in 2002. Journal of gastroenterology. 2007;42(18):6-8.

13. Umehara H, Okazaki K, Nakamura T, Satoh-Nakamura T, Nakajima A, Kawano M, et al. Current approach to the diagnosis of IgG4-related disease-Combination of comprehensive diagnostic and organ-specific criteria. Modern rheumatology. 2017;27(3):381-91.

14. Pathogenesis and clinical manifestations of IgG4-related disease - UpToDate [Internet]. [cited 2021 Mar 16]. Available from: https://www-uptodate-com.ckmproxy.vumc.org/contents/pathogenesis-and-clinical-manifestations-of-igg4related-disease? search=igg4\%20related\%20disease\&source=search_result\&selectedTitle=1 150\&usage_type=default\&display_rank=1

15. Suzuki M, Sai JK, Shimizu T. Acute pancreatitis in children and adolescents. World Journal of Gastrointestinal Pathophysiology. 2014 Nov 15;5(4):416-26.

16. Scheers I, Palermo JJ, Freedman S, Wilschanski M, Shah U, Abu-El-Haija M, et al. Autoimmune Pancreatitis in Children: Characteristic Features, Diagnosis, and Management. Am J Gastroenterol. 2017 Oct;112(10):1604-11.

17. Karim F, Loeffen J, Bramer W, Westenberg L, Verdijk R, van Hagen M, et al. IgG4-related disease: a systematic review of this unrecognized disease in pediatrics. Pediatr Rheumatol Online J. 2016 Mar 25;14(1):18.

18. Inoue D, Yoshida K, Yoneda N, Ozaki K, Matsubara T, Nagai K, et al. IgG4-related disease: dataset of 235 consecutive patients. Medicine (Baltimore). 2015 Apr;94(15):e680.

19. Koizumi S, Kamisawa T, Kuruma S, Tabata T, Chiba K, Iwasaki S, et al. Organ Correlation in IgG4-Related Diseases. J Korean Med Sci. 2015 Jun;30(6):743-8.

20. Wallace ZS, Deshpande V, Mattoo H, Mahajan VS, Kulikova M, Pillai S, et al. IgG4-Related Disease: Clinical and Laboratory Features in One Hundred Twenty-Five Patients. Arthritis Rheumatol. 2015 Sep;67(9):2466-75. 
21. Huang X, Lu B, Li M, Fan Y, Zhang L. IgG4-related retroperitoneal fibrosis overlapping with primary biliary cirrhosis and primary Sjögren's syndrome. Medicine (Baltimore) [Internet]. 2018 Jun 29 [cited 2021 Mar 16];97(26). Available from: https://www.ncbi.nlm.nih.gov/pmc/articles/PMC6039603/

22. Takasumi M, Miyata M, Kuroda M, Terashima K, Abe K, Takahashi A, et al. Overlap of IgG4-related Disease and Primary Biliary Cirrhosis Complicated with Autoimmune Thrombocytopenia. Intern Med. 2016;55(10):1387-92.

23. Kamisawa T, Chen PY, Tu Y, Nakajima H, Egawa N, Tsuruta $K$, et al. Pancreatic cancer with a high serum IgG4 concentration. World J Gastroenterol. 2006 Oct 14;12(38):6225-8.

24. Ngwa T, Law R, Hart P, Smyrk TC, Chari ST. Serum IgG4 elevation in pancreatic cancer: diagnostic and prognostic significance and association with autoimmune pancreatitis. Pancreas. 2015 May;44(4):557-60.

25. Pak LM, Schattner MA, Balachandran V, D’Angelica MI, DeMatteo RP, Kingham TP, et al. The clinical utility of immunoglobulin G4 in the evaluation of autoimmune pancreatitis and pancreatic adenocarcinoma. HPB (Oxford). 2018 Feb;20(2):182-7.

26. Sebastian A, Sebastian M, Misterska-Skóra M, Donizy P, Hałoń A, Chlebicki A, et al. The variety of clinical presentations in IgG4-related disease in Rheumatology. Rheumatol Int. 2018;38(2):303-9.

27. Fong W, Liew I, Tan D, Lim KH, Low A, Leung YY. IgG4-related disease: features and treatment response in a multiethnic cohort in Singapore. Clin Exp Rheumatol. 2018 Jun;36 Suppl 112(3):89-93.

28. Yamada K, Yamamoto M, Saeki T, Mizushima I, Matsui S, Fujisawa Y, et al. New clues to the nature of immunoglobulin G4-related disease: a retrospective Japanese multicenter study of baseline clinical features of 334 cases. Arthritis research \& therapy. 2017;19(1):1-10.

29. Ph L, KI K, Ct H, LI L, Rk T, Tt C, et al. Immunoglobulin G4-related disease in Hong Kong: clinical features, treatment practices, and its association with multisystem disease. Hong Kong Med J. 2017 Sep 1;23(5):446-53.

30. Gupta N, Mathew J, Mohan H, Chowdhury SD, Kurien RT, Christopher DJ, et al. Addition of second-line steroid sparing immunosuppressants like mycophenolate mofetil improves outcome of Immunoglobulin G4-related disease (IgG4RD): a series from a tertiary care teaching hospital in South India. Rheumatol Int. 2018 Feb;38(2):203-9.

31. Chen H, Lin W, Wang Q, Wu Q, Wang L, Fei Y, et al. IgG4-related disease in a Chinese cohort: a prospective study. Scand J Rheumatol. 2014;43(1):70-4.

32. Lin W, Lu S, Chen H, Wu Q, Fei Y, Li M, et al. Clinical characteristics of immunoglobulin G4-related disease: a prospective study of 118 Chinese patients. Rheumatology (Oxford). 2015 Nov;54(11):1982-90.

\section{Tables}

Table 1: Components and Description of Comprehensive Diagnostic Criteria 2011 (Umehara et al) used for classification of study subjects

\begin{tabular}{|c|c|c|c|}
\hline \multicolumn{3}{|c|}{ Components of the Comprehensive Diagnostic Criteria ${ }^{13}$. } \\
\hline Clinical/ Radiological findings & High serum IgG4 & Histopathological findings & \\
& & & \\
\hline Present & Present & Present & Definitive \\
\hline Present & Absent & Present & Probable \\
\hline Present & Present & Absent & Possible \\
\hline
\end{tabular}

Table 2: Classification of study groups I and II according to the Comprehensive Diagnostic Criteria 2011 (Umehara et al) 


\begin{tabular}{|c|c|c|c|}
\hline \multicolumn{3}{|c|}{$\mathbf{n}$} & Diagnosis \\
\hline $\begin{array}{c}\text { Overall } \\
\mathrm{n}=177\end{array}$ & $\begin{array}{c}\text { Pediatric } \\
\mathrm{n}=15\end{array}$ & $\begin{array}{c}\text { Adults } \\
\mathrm{n}=162\end{array}$ & IgG4RD \\
\hline 2 & - & 2 & Definitive \\
\hline 61 & 5 & 56 & Probable \\
\hline 114 & 10 & 104 & Possible \\
\hline
\end{tabular}

Table 3: Pattern of organ involvement in subjects with elevated serum IgG 4 levels

\begin{tabular}{|c|c|c|c|c|c|c|c|}
\hline \multirow[b]{2}{*}{ iagnosis } & \multirow[b]{2}{*}{$\begin{array}{l}\text { Overall } \\
(\mathrm{n}=177)\end{array}$} & \multicolumn{3}{|c|}{ Group I } & \multicolumn{3}{|c|}{ Group II } \\
\hline & & $\begin{array}{c}\text { All } \\
\text { subjects } \\
(\mathrm{n}=115)\end{array}$ & $\begin{array}{l}\text { Pediatric } \\
\text { Population } \\
(\mathrm{n}=10)\end{array}$ & $\begin{array}{c}\text { Adult } \\
\text { Population } \\
(\mathrm{n}=105)\end{array}$ & $\begin{array}{c}\text { All } \\
\text { subjects } \\
(\mathrm{n}=62)\end{array}$ & $\begin{array}{c}\text { Pediatric } \\
\text { Population } \\
(\mathrm{n}=05)\end{array}$ & $\begin{array}{c}\text { Adult } \\
\text { Population } \\
(n=57)\end{array}$ \\
\hline & n (\%) & n (\%) & n (\%) & n (\%) & n (\%) & n (\%) & n (\%) \\
\hline ancreas & $102(57.6)$ & $86(74.7)$ & $7(70)$ & $79(75.2)$ & $16(25.8)$ & - & $16(28)$ \\
\hline $\begin{array}{l}\text { ubmandibular } \\
\text { land }\end{array}$ & $22(12.4)$ & $5(4.3)$ & $2(20)$ & $3(2.8)$ & $17(27.4)$ & - & 17(29.8) \\
\hline iver & $11(6.2)$ & $11(9.5)$ & - & $11(10.4)$ & & - & - \\
\hline$\overline{\text { yes }}$ & $9(5)$ & - & - & - & $9(14.5)$ & $4(80)$ & $5(8.8)$ \\
\hline all bladder & $8(4.5)$ & $3(2.6)$ & - & $3(2.8)$ & $5(8)$ & - & $5(8.8)$ \\
\hline$\overline{\text { ung }}$ & $8(4.5)$ & $1(0.8)$ & - & $1(0.9)$ & $7(11.2)$ & - & $7(12.3)$ \\
\hline ymph node & $8(4.5)$ & $3(2.6)$ & $1(10)$ & $2(1.9)$ & $5(8)$ & - & $5(8.8)$ \\
\hline hyroid & $3(1.6)$ & $2(1.7)$ & - & $2(1.9)$ & $1(1.6)$ & - & $1(1.8)$ \\
\hline idney & $2(1.1)$ & $2(1.7)$ & - & $2(1.9)$ & & - & - \\
\hline enis & $1(0.5)$ & - & - & - & $1(1.6)$ & $1(20)$ & - \\
\hline acrimal gland & $1(0.5)$ & - & - & - & $1(1.6)$ & - & $1(1.7)$ \\
\hline lood Vessels & $1(0.5)$ & $1(0.8)$ & - & $1(0.9)$ & - & - & - \\
\hline $\begin{array}{l}\text { etroperitoneal } \\
\text { brosis }\end{array}$ & $1(0.5)$ & $1(0.8)$ & - & $1(0.9)$ & - & - & - \\
\hline
\end{tabular}

Table 4: Studies Reported from Asia on IgG4 Related Disease using Comprehensive Diagnostic Criteria (from 2014 to 2019) 


\begin{tabular}{|l|l|l|l|l|l|}
\hline $\begin{array}{c}\text { Reference } \\
\text { number }\end{array}$ & \multicolumn{1}{|c|}{ Authors } & Year & Country & $\begin{array}{c}\text { Subjects } \\
\text { (n) }\end{array}$ & \multicolumn{1}{|c|}{$\begin{array}{c}\text { Most commonly affected } \\
\text { organs }\end{array}$} \\
\hline- & Current Study & 2019 & Pakistan & 114 & $\begin{array}{l}\text { Pancreas 57.6\% } \\
\text { Submandibular gland 12.4\% } \\
\text { Liver 6.2\% }\end{array}$ \\
\hline 27 & Fong, W. et al & 2018 & Singapore & 42 & $\begin{array}{l}\text { Pancreas 65\% } \\
\text { Lymph node 45\% } \\
\text { Hepatobiliary 40\% }\end{array}$ \\
\hline 28 & $\begin{array}{l}\text { Yamada Kazunori et } \\
\text { al* }\end{array}$ & 2017 & Japan & 334 & $\begin{array}{l}\text { Salivary gland 72.7\% } \\
\text { Lacrimal gland 57.1\% } \\
\text { Lymph node 56.5\% }\end{array}$ \\
\hline 30 & Ph L et al & 2017 & $\begin{array}{l}\text { Hong } \\
\text { Kong }\end{array}$ & 104 & $\begin{array}{l}\text { Hepatobiliary and pancreas } \\
40.4 \% \\
\text { Salivary gland 33.7\% } \\
\text { Lymph node 29.8\% }\end{array}$ \\
\hline 30 & 2016 & India & 41 & $\begin{array}{l}\text { Eye 13\% } \\
\text { Lymph node 11\% } \\
\text { Retroperitoneum 11\% }\end{array}$ \\
\hline 31 & Chen H et al & 2014 & China & 28 & $\begin{array}{l}\text { Salivary gland 79\% } \\
\text { Lacrimal gland 46\% } \\
\text { Lymph node 43\% }\end{array}$ \\
\hline 32 & Lin W et al & 2015 & China & 118 & $\begin{array}{l}\text { Lymph node 65.3\% } \\
\text { Salivary gland 64.4\% } \\
\text { Lacrimal gland 50.8\% }\end{array}$ \\
\hline
\end{tabular}

\section{Figures}


Figure 1a: Symptoms in pediatric patients in Group I.



Figure 1b: Symptoms in adult patients in Group 1

70



Figure 1

Symptomatology in subjects with elevated serum IgG4 (Group I) tested at AKUH Clinical Laboratory. Figure 1 a describes symptoms in ten children ( $<18$ years of age) with high serum IgG4 while figure $1 \mathrm{~b}$ describes one hundred and five adults who had high IgG4. Figure 1b: Symptoms in adult patients with high serum IgG4 levels. 